\title{
Demonstration of the evolution of spectral resolving power as a superposition of higher order delayed beams
}

Chandrasekhar Roychoudhuri, Tariq Manzur

Chandrasekhar Roychoudhuri, Tariq Manzur, "Demonstration of the evolution of spectral resolving power as a superposition of higher order delayed beams," Proc. SPIE 2525, 1995 International Conference on Education in Optics, (13 October 1995); doi: 10.1117/12.224025

Event: SPIE's 1995 International Symposium on Optical Science, Engineering, and Instrumentation, 1995, San Diego, CA, United States 


\title{
DEMONSTRATION OF THE EVOLUTION OF SPECTRAL RESOLVING POWER AS A SUPERPOSITION OF HIGHER ORDER DELAYED BEAMS
}

\author{
Chandra Sekhar Roychoudhuri and Tariq Manzur
}

\author{
Photonics Research Center \& Electrical \& Systems Engineering \\ University of Connecticut, 260 Glenbrook Rd, U-157, Storrs, CT 06269 \\ Phone: (203) 486-2587, Fax: (203) 486-1033, E-Mail: chandra@eng2.uconn.edu
}

\begin{abstract}
Introductory textbooks on basic optics introduce gratings and Fabry-Perot spectrometers separately with the logic that they are based on (i) diffraction and interference or (ii) division of wavefront and division of amplitude. Since in modern classrooms, most of the experiments can be carried out with highly coherent and collimated laser beams, we propose to introduce that gratings and Fabry-Perots are equivalent to each other by using a "pencil" He-Ne beam illuminating a plane-parallel Fabry-Perot at an angle. It produces a set of distinct and parallel pencil of beams by multiple reflection resembling a multiple slit illumination ${ }^{1}$. When these beams are superposed by a focusing lens, a multiple slit diffraction grating like pattern is observed. One can use an appropriately spaced etalon and reflectivity to demonstrate the evolution of resolving power by allowing step-by-step an increasing number of beams to interfere at the focus and slowly resolve the longitudinal modes (or frequencies) of the He-Ne laser.
\end{abstract}

\section{INTRODUCTION}

The interference between two or more, beams replicated from the original beam, with a specific path delay between the beams is at the root of all spectral resolving power. This is true for spectrometers which can be represented by three basic groups: (i) Two beam Michelson's (Fourier transform) spectrometer, (ii) Multiple-beam Fabry-Perot spectrometer and (ii) Multiple beam grating spectrometer. The first two category can be classified as of the type "amplitude division" as the entire wave front is replicated through amplitude division by partial mirror (s) and the gratings can be classified as of the type "wave front division" as the wave front is divided into multitudes of secondary wave fronts by the grating lines for ultimate interference. In this paper we simulate the grating like behavior of a plane parallel Fabry-Perot spectrometer using a narrow but 
collimated laser beam illuminating the Fabry-Perot at an angle. This approach reveals the similarity between Fabry-Perots and gratings as spectral instruments. It also helps one to appreciate why interference of high order and superposition of a large number of beams complement each other to achieve high spectral resolution. The order of interference is the optical path difference between the consecutive interfering beams expressed in number of wavelengths. The separation between the neighboring fringe maxima for a two closely spaced frequencies will be larger for larger path delay (or larger order of interference). The frequencies will be well resolved when the number of interfering beams is large enough that the width of the main fringe peak for each frequency is narrower than the spacing between the peaks for the two frequencies. A plane grating achieves high resolution by increasing the total number of beams by virtue of increasing the number of grating lines. But a Fabry-Perot achieves its high resolution by increasing both the order of interference by virtue of increasing plate separation and the effective number of interfering beams by virtue of increasing reflectivity (reflective finesse). Our objective is to demonstrate these concepts graphically and experimentally using a FabryPerot Spectrometer.

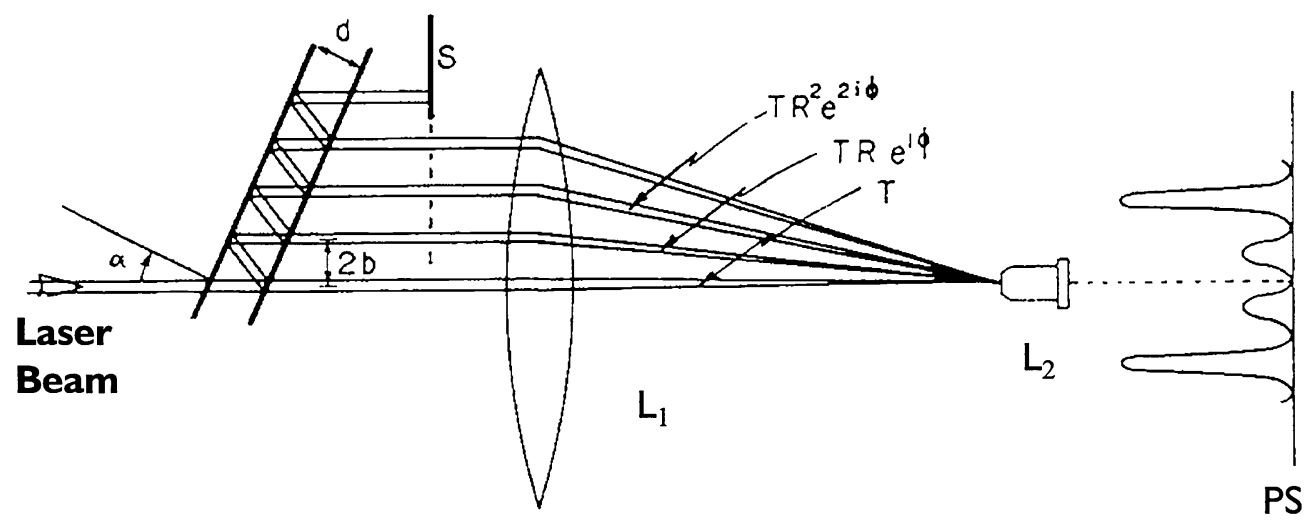

Fig. I. Experimental setup to demonstrate that: (i) increase in spectral resolution is a product of the increasing order of interference and the increasing number of the interfering beams, and (ii) a Fabry-Perot and a grating are equivalent on this fundamental level. FP, Fabry-Perot; S, Screen to control the number of beams; $L_{1}$, focusing lens; $L_{2}$, microscope objective; PS, multiple-beam fringes on the projection screen '. 


\section{EXPERIMENTAL SETUP}

A narrow light beam straight from a laser (Fig. 1) is incident on one end of the FP etalon at an angle such that the beams generated by multiple reflections are spatially separated from each other as if a multiple-slit screen or, more appropriately, an echelle grating had been placed in front of an extended collimated beam. At the focal plane of the lens $\mathrm{L}_{1}$, immediately following the FP, one can see interference fringes which have properties similar to the multiple-slit interference pattern. For convenience of observation by many people, the fringes can be projected on a screen by a microscope objective.

During experimental set up, the lenses $\mathrm{L}_{1}$ and $\mathrm{L}_{2}$ are adjusted to be coaxial with the laser (which can be tested by observing the beams reflected from the lens surfaces) to minimize optical aberration. Then the FP etalon is inserted at an angle to the axis before $\mathrm{L}_{1}$, preferably on a turntable, to control the angle of insertion. This control over the angle of insertion will control the lateral separation between the transmitted beams produced by multiple reflection. Thus, a continuous tilt of the etalon will give continuous change in the separation between the "slits". If a two plate FP interferometer is used instead of an etalon, the parallelism between the plates can be easily achieved by first allowing the incident beam to be perpendicular to the first mirror and then adjusting the other mirror until all the transmitted beams are exactly coincident and gives high finesse Fabry-Perot fringes when one of the mirrors is scanned.

The effect of changing the number of beams or "grating slits" can now be easily demonstrated by moving a screen S (Fig. 1) to allow as many beams as are wanted.

\section{THEORY}

The mathematical expression for such a multiple-beam interference pattern with a FP can be derived from its similarity to a diffraction grating. The only difference is the decrease in amplitude of the consecutive beams and the increase in path difference due to their traveling through the FP. In Fig. 1, the consecutive beams are shown to have a separation of $2 \mathrm{~b}$ with the appropriate changes in amplitudes and phases. We shall avoid using the Fourier transformation technique by using a simplified model: a plane wave incident on a lens produces a small "spot" of plane wave at the focal plane of the lens (focal spot) where the irradiance distribution follows the Fraunhofer diffraction pattern for a single 
"slit", in this case, the laser beam diameter. Then the situation of many plane waves incident on a lens can be visualized in Fig. 2. (The size of the focal spot is exaggerated).

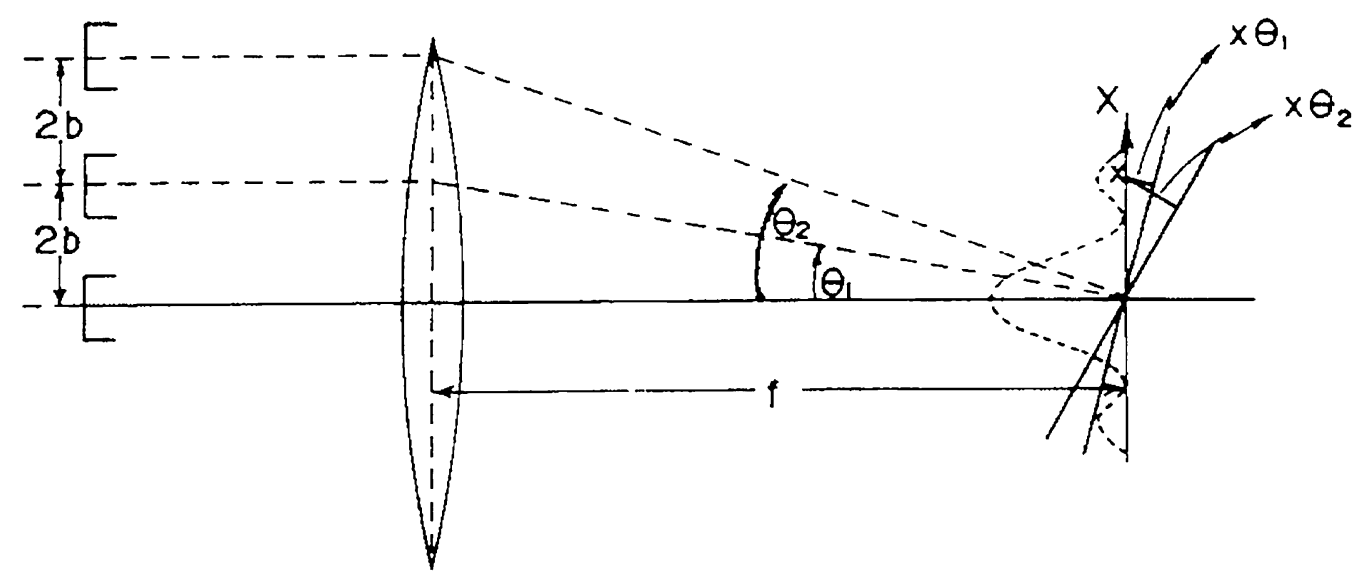

Fig. 2 Multiple-slit Fraunhofer diffraction pattern as interference between plane-wave "spots" with regularly increasing tilt at the focal plane.

The successive plane waves generated at the focal plane of the lens interfere with each other at angles

$$
\theta_{n} \approx \tan \theta_{n} \approx \sin \theta_{n} \approx n(2 b / f),
$$

where $\mathrm{n}$ is an integer and $\mathrm{f}$ is the focal length of the lens $\mathrm{L}_{1}$. So, the phase difference at a point $\mathrm{x}$ is,

$$
\phi_{n}=k x \sin \theta_{n} \approx n(2 k b x / f) \equiv n \phi \equiv n k \delta_{1}
$$

where

$$
k=2 \pi / \lambda \text {. }
$$

In our case, the amplitude and phase of the successive beams are also modified by the Fabry-Perot etalon. So the n-th wavefront can be written as

$$
T R^{n} \exp (i n \psi)
$$


where $\mathrm{T}$ and $\mathrm{R}$ are the intensity transmittance and reflectance, respectively, and $\Psi$ is the optical path delay between any two consecutive beams, given by,

$$
\Psi=k(2 d p \lambda \cos \alpha) \equiv k \delta_{2},
$$

where $d$ and $\alpha$ are shown in Fig. 1, and $\rho$ is the refractive index of the medium between the two reflecting surfaces. We shall neglect any constant phase shift introduced by the reflecting surface. So, the resultant amplitude of interference for our case is

$$
A=\sum_{n=0}^{N-1} T R^{n} \exp (\operatorname{in} \psi) \exp (i n \phi)
$$

Then the irradiance distribution is,

$$
\mathbf{I}=\mathrm{T} \frac{1+\mathbf{F} \mathbf{N} \sin ^{2} \mathbf{N}(\psi+\phi) / 2}{1+\mathbf{F} \sin ^{2}(\psi+\phi) / 2}
$$

where

$$
\begin{aligned}
& \mathrm{T} \equiv T^{2}\left(1-R^{N}\right)^{2} /(1-R)^{2}, \\
& \mathbf{F}_{\mathbf{N}} \equiv 4 \mathbf{R}^{\mathbf{N}} /\left(1-\mathbf{R}^{\mathbf{N}}\right)^{2},
\end{aligned}
$$

and

$$
F \equiv 4 R /(1-R)^{2}
$$

Equation (6) will also describe the fringe pattern for a Lummer-Gehrcke plate. This suggests that the demonstration experiment can also be carried out by using a LummerGehrcke plate, if available. Equation (5) will give an exact FP Airy function in the limit when $\mathrm{N}$ tends to infinity and the separation between the "slits" goes to zero $(2 \mathrm{~b}=0$, when $\Phi=0$ ) The same equation becomes identical with the ordinary diffraction grating equation when one replaces $T^{\mathrm{n}}$ by unity and $\Psi$ by zero. In the above derivation, we have neglected the small changes in phase curvature and the beam waists of the Gaussian laser beams due its propagation. 
If a He-Ne laser oscillates in $\mathrm{q}$ independent modes, the spectral intensity distribution of Equation 6 should be summed over all the q-modes with appropriate intensities $I_{q}$ (Fig. 3),

$$
\mathrm{I}=T \sum_{q} I q \frac{1+F_{N} \sin ^{2} N(\psi+\phi) / 2}{1+F \sin ^{2}(\psi+\phi) / 2}
$$

\section{ENERGY}

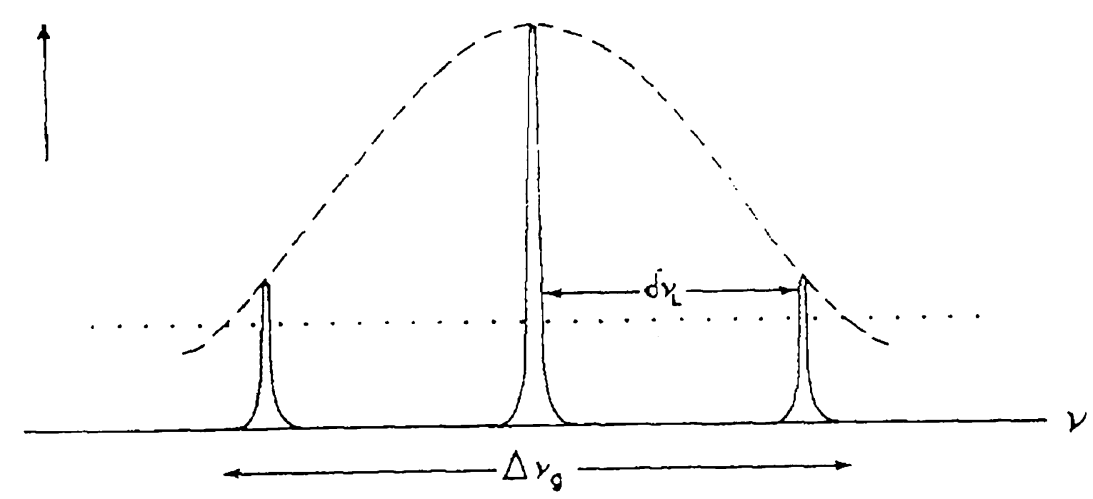

Fig. 3 Longitudinal modes of a laser. $\Delta v_{g}$, gain bandwidth of the lasing medium; $\delta v_{L}$, the separation between two consecutive longitudinal modes. The dotted line corresponds to the lasing threshold.

\section{COMPUTER SIMULATION AND EXPERIMENTAL RESULTS}

Let us consider the specific case of a $30 \mathrm{~cm}$ cavity He-Ne Laser oscillating dominantly in 3 modes ( $\mathrm{q}=3$ ) around mean wavelength of $6328 \AA$ with a mode spacing of $500 \mathrm{MH}_{\mathrm{Z}}$ (or $0.06674 \AA$ ). The plane parallel FP we have used, has mirrors of $\mathrm{R}_{1}=\mathrm{R}_{2}=0.98$ separated by air $(\rho=1)$. The resolving power for a grating is the product of the order of interference, $m$, and the total nuthber of grating lines, $N$, intercepted by the spatially coherent light beam. We will demonstrate that the resolving power of our tilted FabryPerot spectrometer is approximately given by the same product of the $\mathrm{MN}$, where $\mathrm{m}$ is $\left(\delta_{1}+\delta_{2}\right) / \lambda$ and $N$ is the number of interfering beams.

Table 1 shows the number of minimum beams $\mathrm{N}_{X}$ necessary for resolving the $\mathrm{He}-\mathrm{Ne}$ modes for several different mirror spacing from about $0.1 \mathrm{~cm}$ to $9 \mathrm{~cm}$. It is clear that as the mirror spacing or equivalently, the interference order number $\mathrm{m}$ is increased, the 
required number of interfering beams $\mathrm{N}$ is reduces. This is because the separation between the $\mathrm{m}$-th order fringes for three modes is larger for larger mirror spacing, requiring less sharper fringes to resolve them.

Above results are demonstrated by experimental and computer plotted graphs in the following nine pages. We have plotted three sets of spectral curves for $d=5,7.5$ and 9 $\mathrm{cm}$. For each case of $\mathrm{d}$, we show the fringe narrowing due to increasing number of interfering beams. Notice in the computer graphics that the separation between the fringe maxima from the three He-Ne modes increases with increasing mirror spacing allowing mode resolution with a fewer number of interfering beams.

\section{ACKNOWLEDGMENTS}

The authors gratefully acknowledge partial support for this work by the Connecticut Innovation Incorporated through the Critical Technologies Grant and by NSF through the program National Alliance for Photonics Education in Manufacturing or NAPEM (Program \# ELS 94-14669). We also appreciate computational support from Jim Anderson and Ken Chen.

\section{REFERENCE}

1. C. Roychoudhuri and R.H. Noble "Demonstrations using a Fabry-Perot. I. Multipleslit interference and II. Laser mode display:; Am. J. Phys. 43 (12), 1054 and 1057 (1975). 


\section{TABLE 1. Evaluation of the Resolving Power With the Minimum Number of Beams For a Given Etalon Spacing}

\begin{tabular}{|c|c|c|c|c|}
\hline $\begin{array}{c}\text { Etalon } \\
\text { Spacing } \\
\text { d } \\
(\mathrm{cm})\end{array}$ & $\begin{array}{l}\text { Interference Order } \\
\mathrm{m}_{\lambda_{\mathrm{o}}} \\
\lambda_{\mathrm{o}}=6328 \stackrel{\mathrm{o}}{\mathrm{A}}\end{array}$ & $\begin{array}{c}\delta \mathrm{m} \\
\text { for the case } \\
\delta v=5 \times 10^{8} \mathrm{~Hz} \\
(30 \mathrm{~cm} \mathrm{He}-\mathrm{Ne})\end{array}$ & $\begin{array}{c}\text { Minimum } \\
\text { resolving } \\
\text { power } \\
\text { needed } \\
\Re \\
\frac{\mathrm{m}}{\delta \mathrm{m}} \times 10^{6} \\
\end{array}$ & $\begin{array}{l}\text { Minimum } \\
\text { no. of Beams } \\
N_{a} \\
=\frac{1}{\delta m}\end{array}$ \\
\hline 0.938 & 295174 & 0.031131 & 0.9482 & 32 \\
\hline 1.875 & 59035 & 0.062262 & 0.9482 & 16 \\
\hline 3.75 & 118070 & 0.124524 & 0.9482 & 8 \\
\hline 5.00 & 157426 & 0.166032 & 0.9482 & 6 \\
\hline 7.50 & 236139 & 0.249048 & 0.9482 & 4 \\
\hline 9.00 & 283367 & 0.298858 & 0.9482 & 3 \\
\hline
\end{tabular}

Resolving power:

$\Re=\frac{\lambda}{\delta \lambda}=\frac{\nu}{\delta \nu}=\frac{m}{\delta m}=\mathrm{m} \mathrm{N} \quad$ ( for grating $\mathfrak{R}=0.9$ ) 

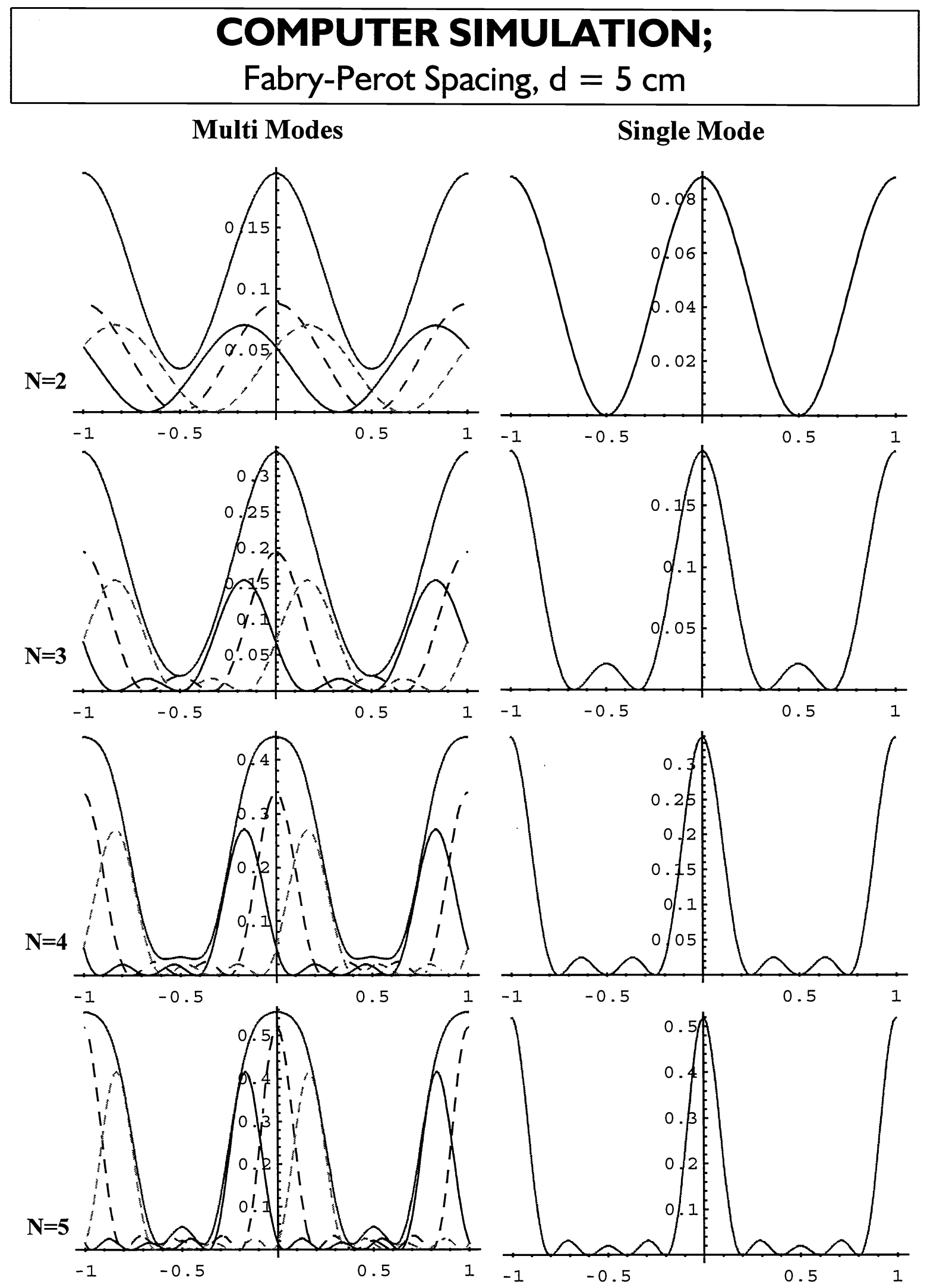

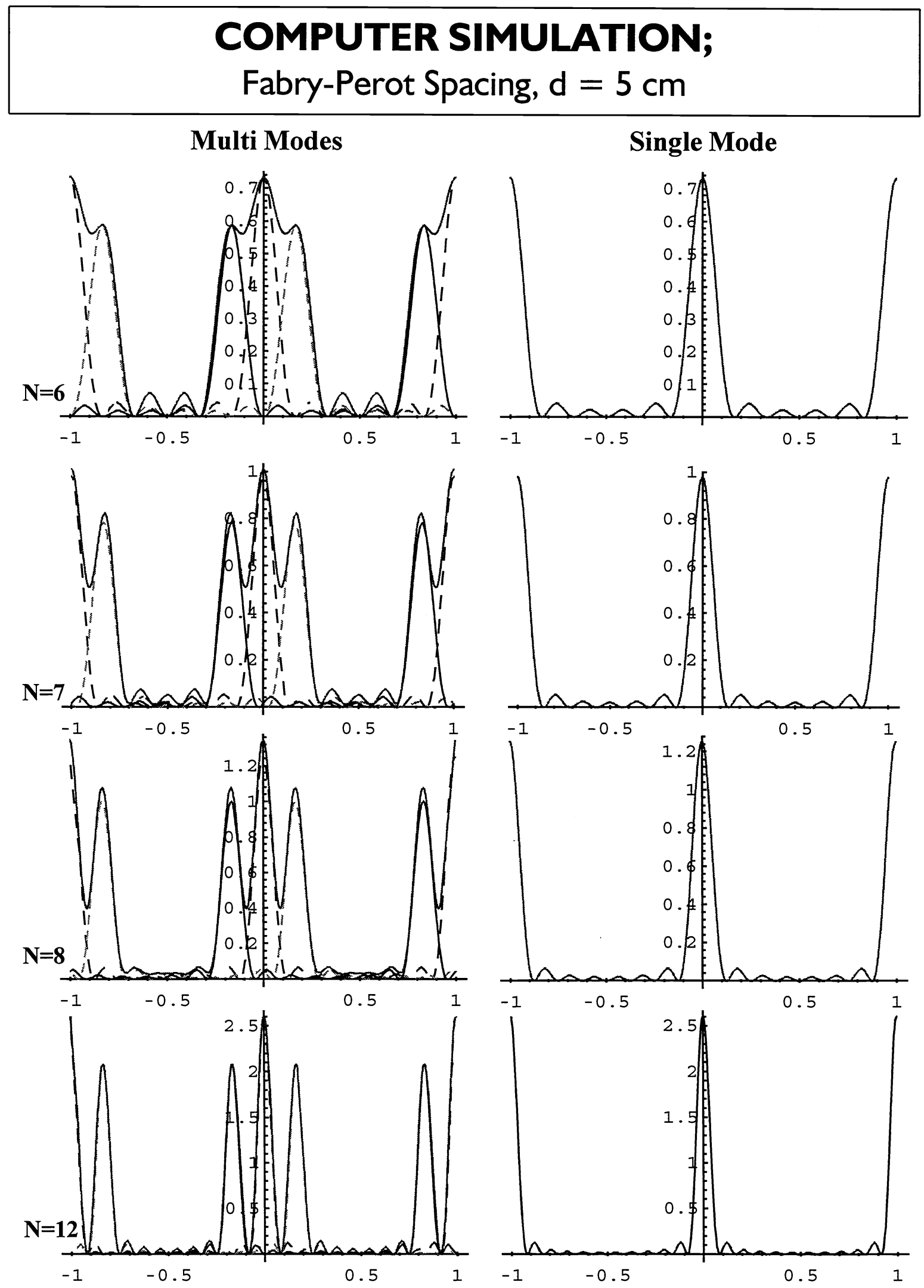


\begin{tabular}{l}
\hline COMPUTER SIMULATION; \\
Fabry-Perot Spacing, $d=7.5 \mathrm{~cm}$ \\
\hline
\end{tabular}

\section{Multi Modes}
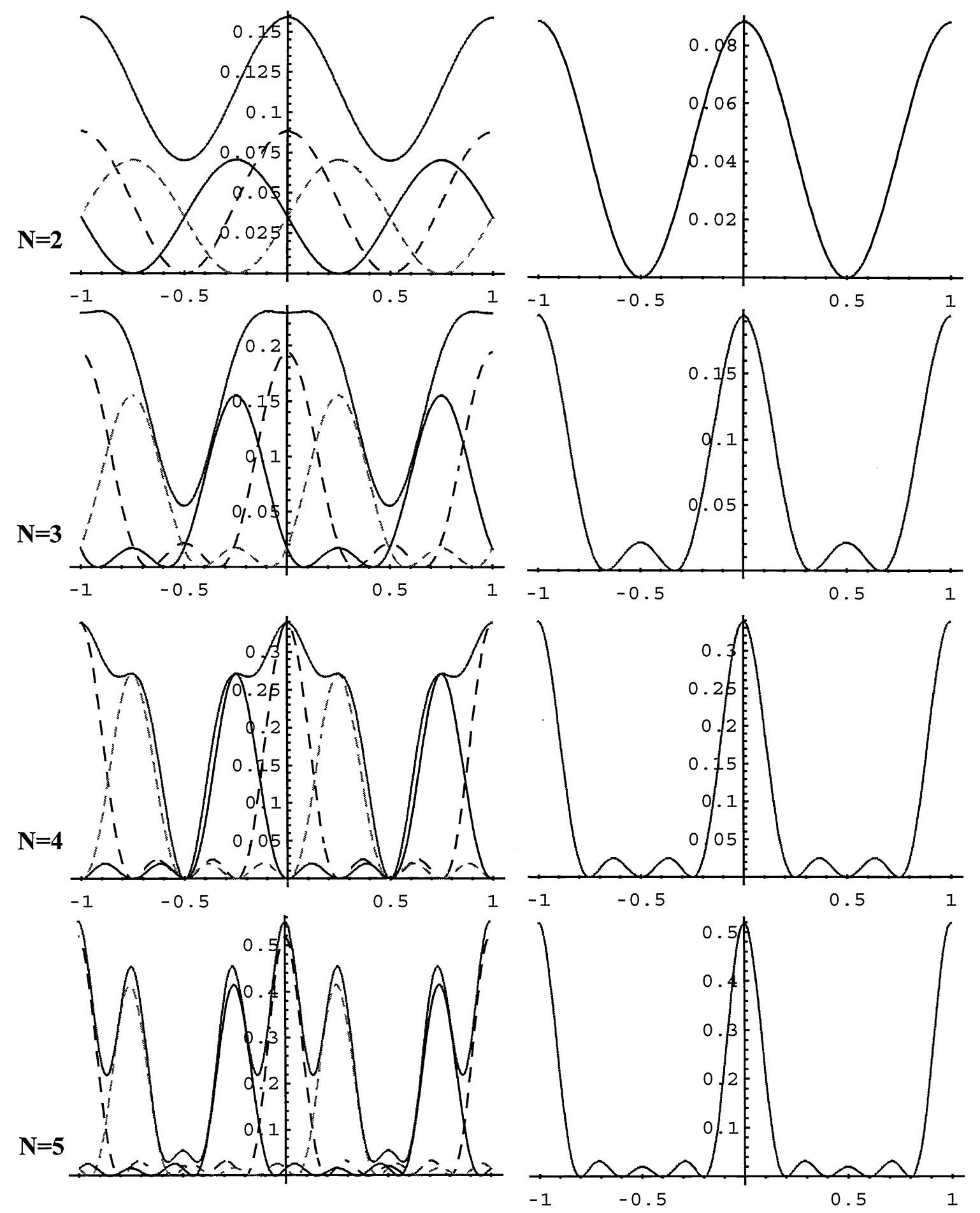


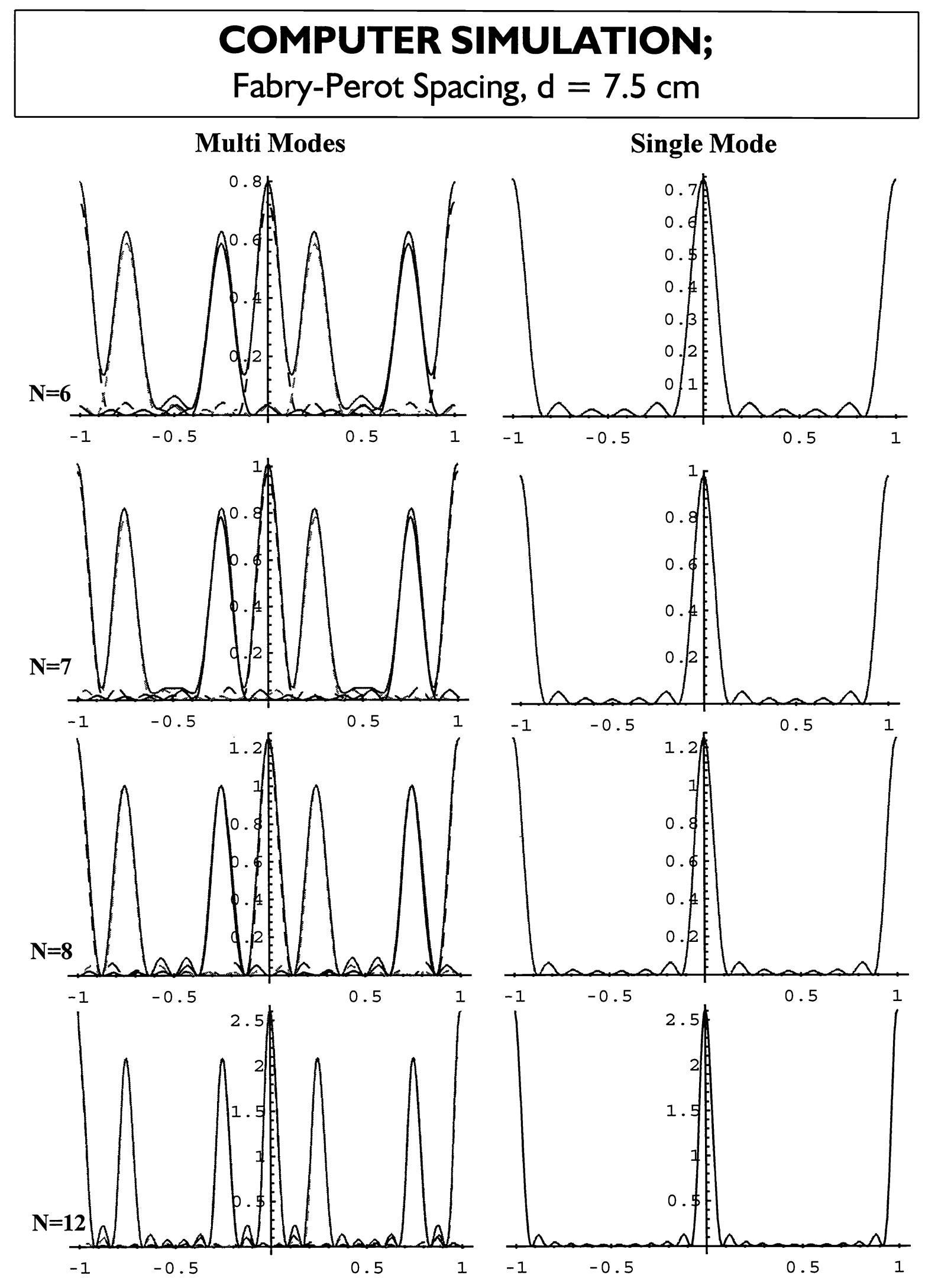

SPIE Vol. $2525 / 39$ 


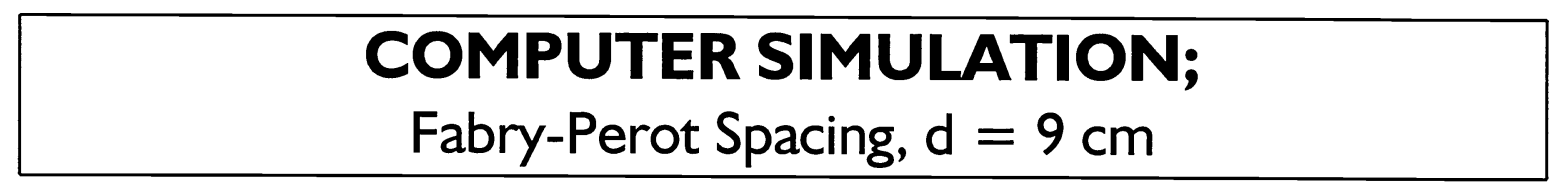

\section{Multi Modes}
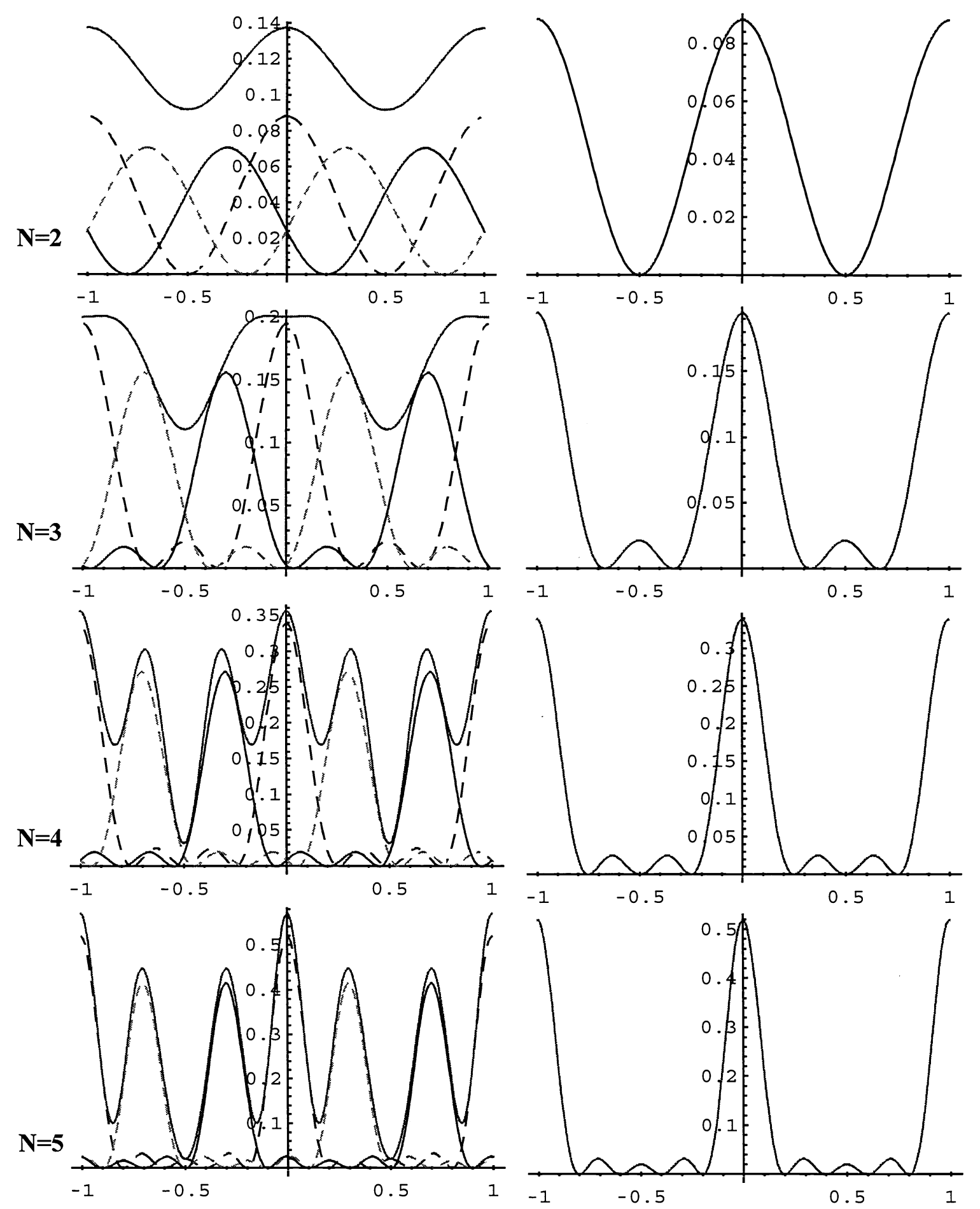

40 / SPIE Vol. 2525 

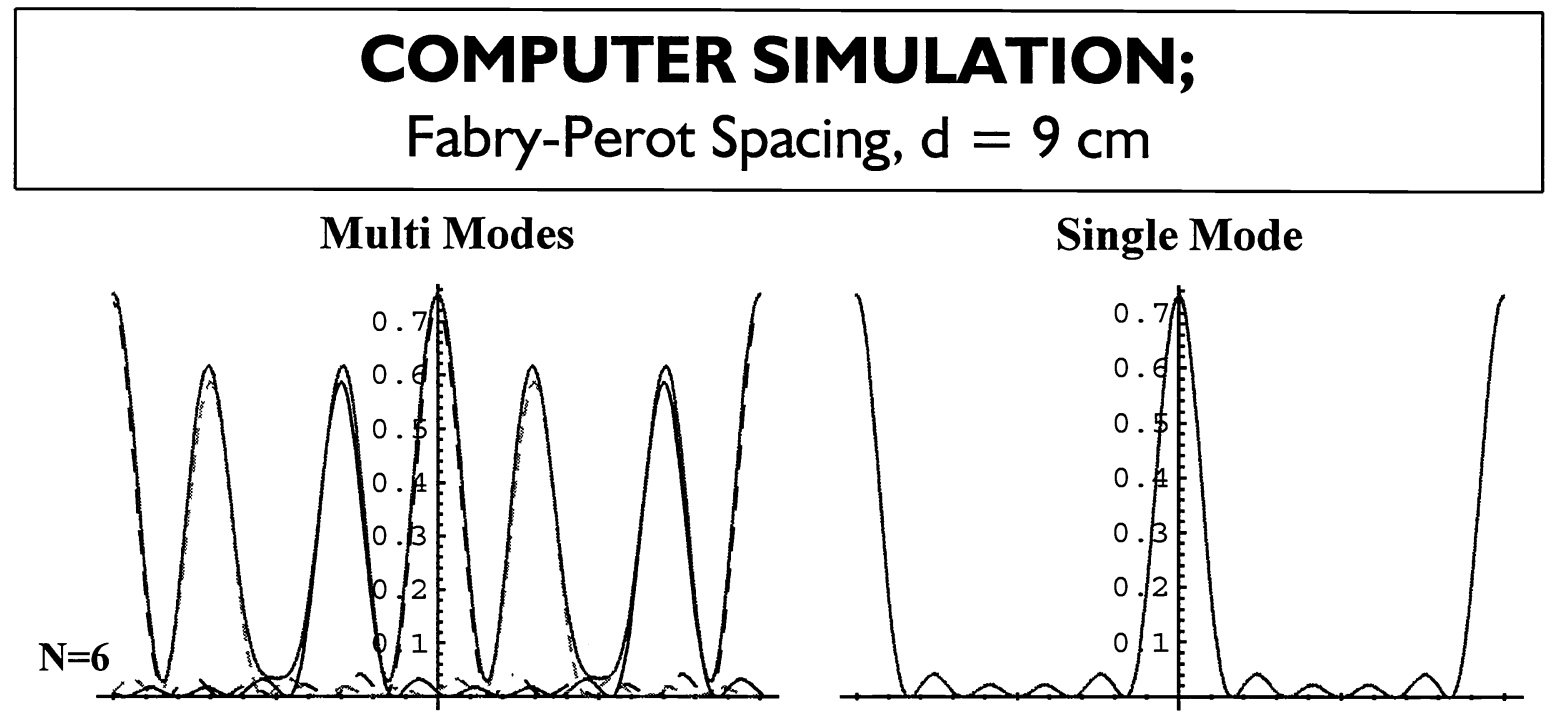

\section{Single Mode}
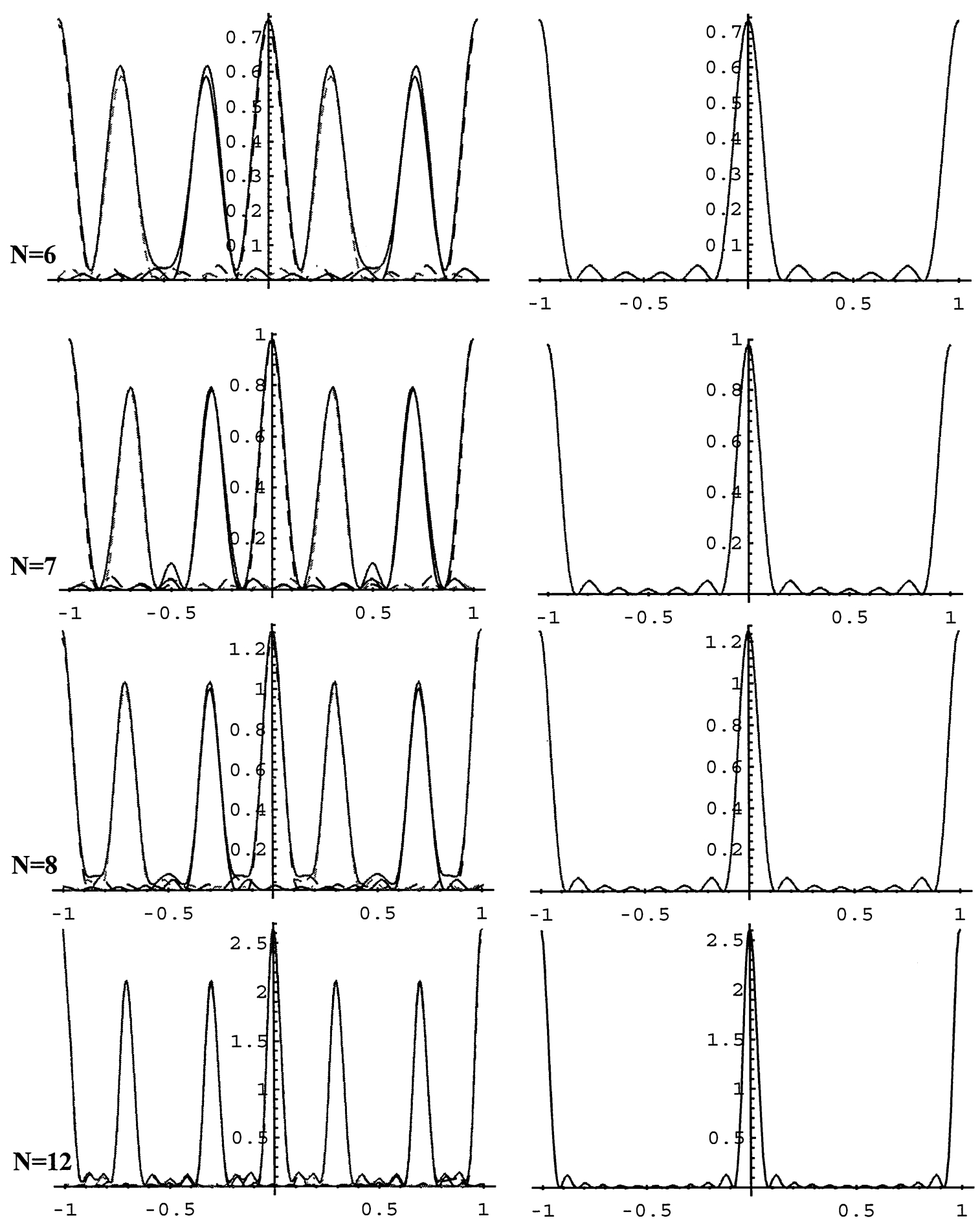


\section{EXPERIMENTAL RESULTS; FP Spacing, $d=5 \mathrm{~cm}$}
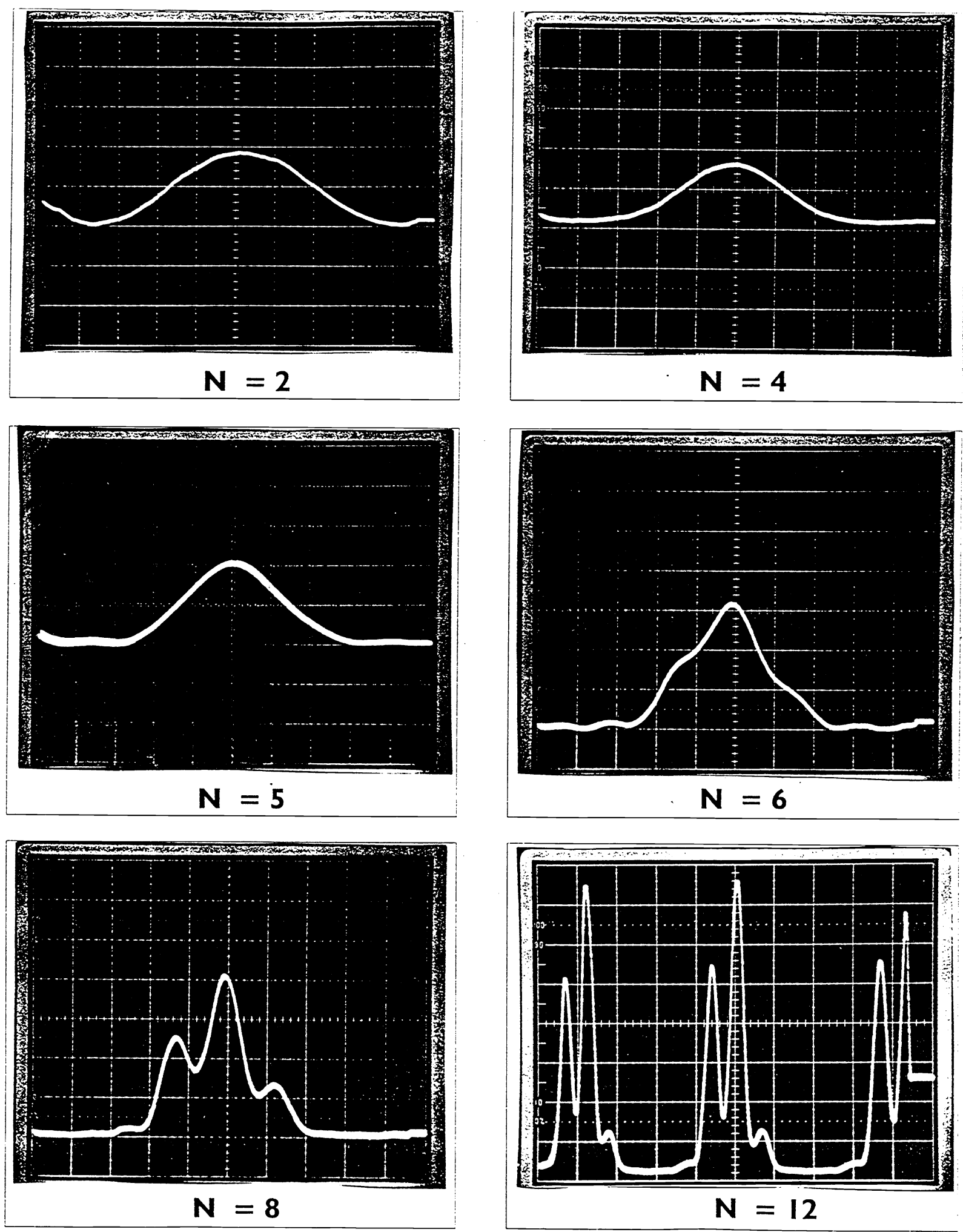


\section{EXPERIMENTAL RESULTS; FP Spacing, $d=7.5 \mathrm{~cm}$}
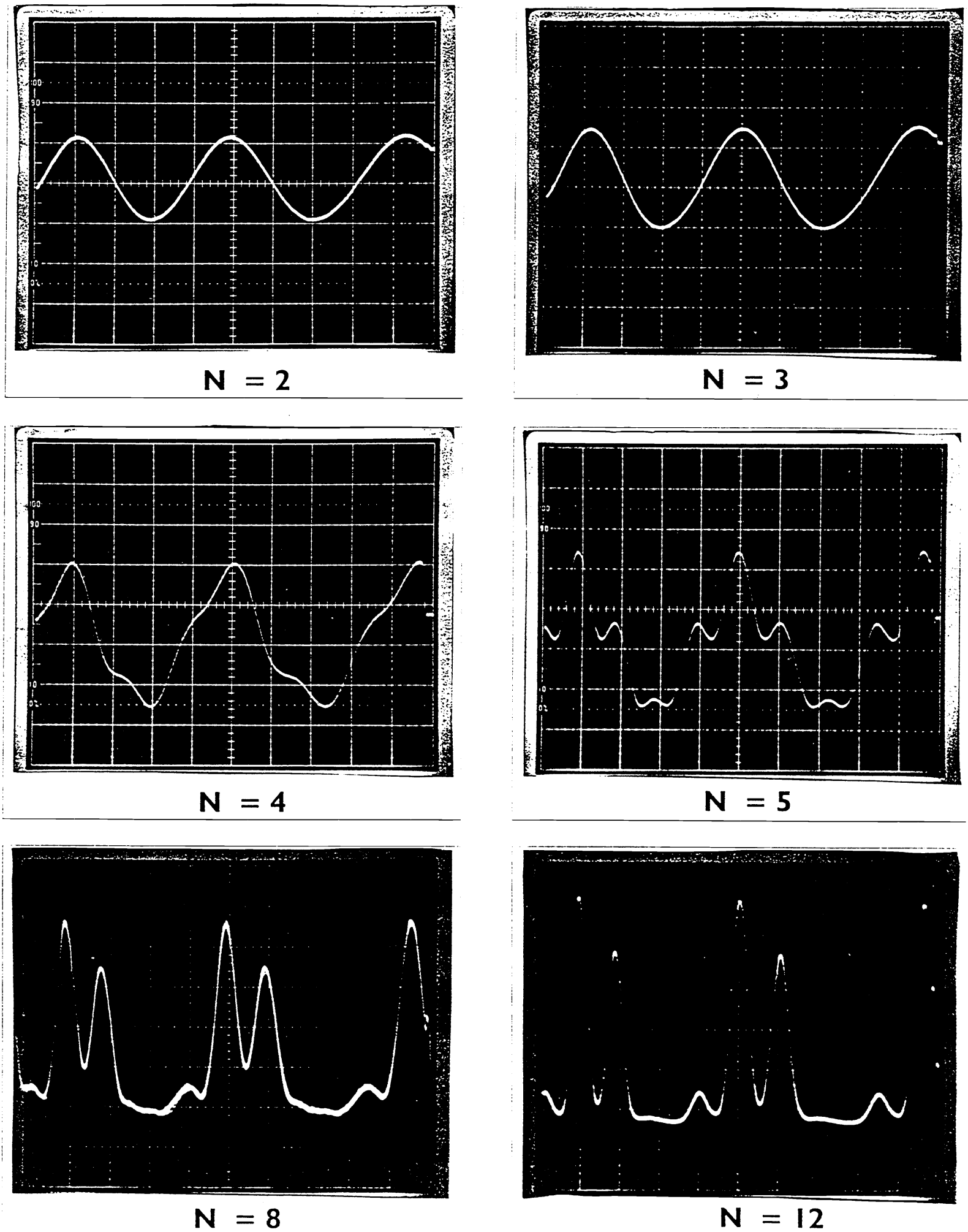


\section{EXPERIMENTAL RESULTS; FP Spacing, $d=9 \mathrm{~cm}$}
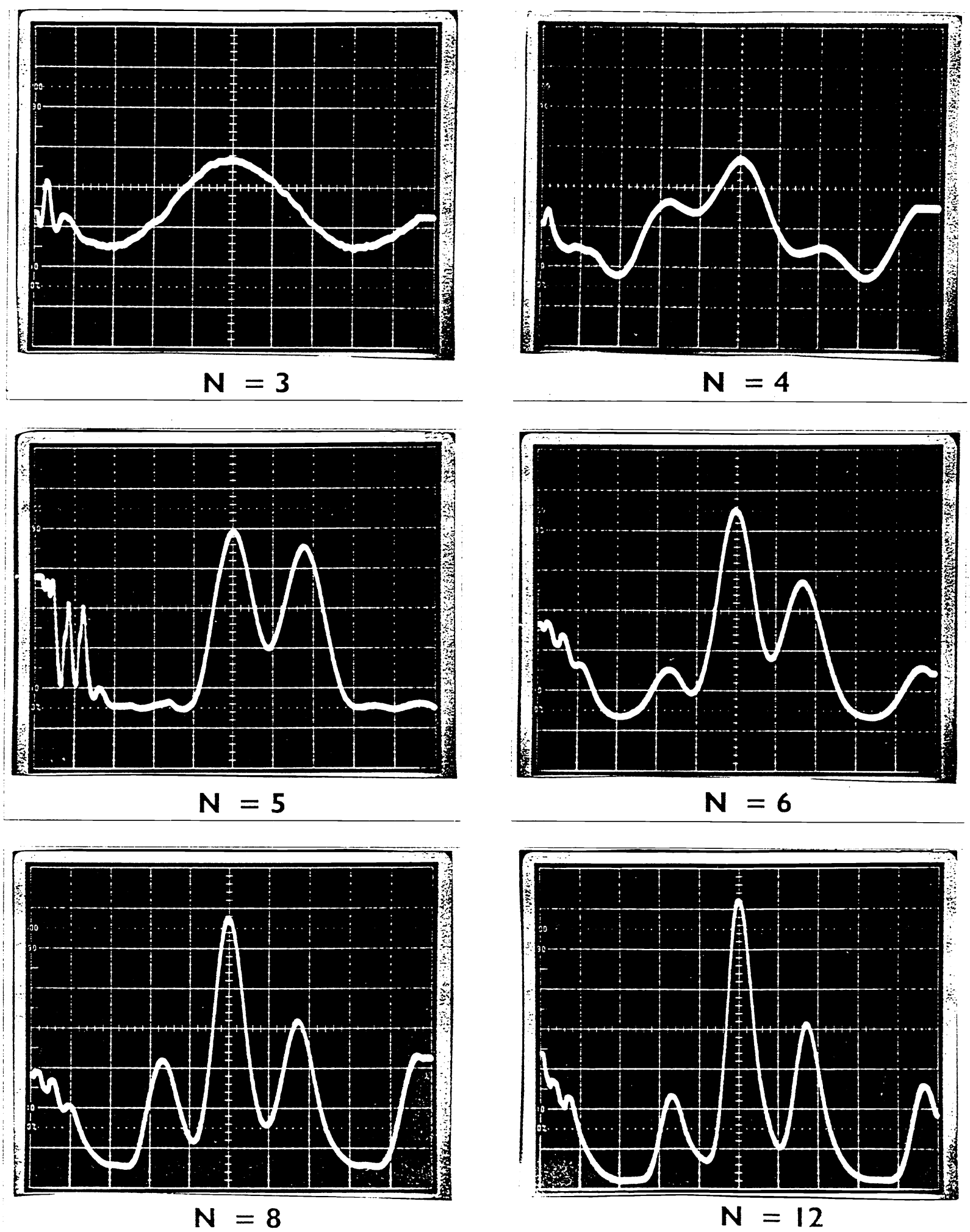